\title{
The Impact of Chronic Obstructive Pulmonary Disease and Hospital Teaching Status on Mortality, Cost, and Length of Stay in Elective Total Hip Arthroplasty Patients
}

Cameron G. Hanson ${ }^{1}$, Kyle L. Barner ${ }^{2}$, Zakary Rose-Reneau ${ }^{3}$, Michael Kortz ${ }^{1}$

1. Miscellaneous, Kansas City University of Medicine and Biosciences, Kansas City, USA 2. Orthopedic Surgery, North Kansas City Hospital, Kansas City, USA 3. Anatomy, Kansas City University of Medicine and Bioscience, Kansas City, USA

Corresponding author: Cameron G. Hanson, cameron.g.hanson@gmail.com

\section{Abstract}

\section{Introduction}

Total hip arthroplasty (THA) is a frequently performed surgery. Chronic obstructive pulmonary disease (COPD) is one of the most prevalent diseases in the United States and has been associated with higher complications in many orthopedic surgeries. The purpose of this study was to examine the clinical and economic impacts of COPD on the mortality, cost, and length of stay of those undergoing THA and the effect of hospital teaching status on these outcomes.

\section{Methods}

This retrospective cohort study identified adult patients ( $\geqslant 18$ years) utilizing information from the Healthcare Cost and Utilization Program Nationwide Inpatient Sample (NIS) from 2012 to 2014 undergoing elective THA using ICD-9 codes. Patients missing key clinical identifiers or those who did not undergo THA were excluded. Mortality, cost, and length of stay were assessed. The COPD cohort was further analyzed by hospital teaching status, including urban teaching, urban non-teaching, and rural.

\section{Results}

An adjusted total of 7,652 patients with COPD and 768,000 patients without COPD undergoing THA were identified. COPD was associated with higher mortality rates, longer lengths of stay, and total charges. In the COPD cohort, teaching status did affect outcomes. Between urban teaching hospitals and urban non-teaching, chronic conditions were significantly higher in urban teaching hospitals, yet total charges were lower. LOS was longer in rural hospitals, however, all other variables, including costs, were not significantly different as compared to urban teaching hospitals. Between urban non-teaching hospitals and rural hospitals, the number of chronic conditions and LOS were higher in rural hospitals, yet costs were significantly less. Age and mortality rates were not significantly different between different teaching statuses.

\section{Conclusion}

COPD has a significant effect on mortality, length of stay, and cost in patients undergoing THA. Additionally, teaching status seems to play an interesting role in these

\section{How to cite this article}

Hanson C G, Barner K L, Rose-Reneau Z, et al. (April 12, 2019) The Impact of Chronic Obstructive Pulmonary Disease and Hospital Teaching Status on Mortality, Cost, and Length of Stay in Elective Total Hip Arthroplasty Patients. Cureus 11(4): e4443. DOI 10.7759/cureus.4443 
variables. Preoperative planning may help surgeons mitigate some of these risks associated with COPD. Further work on how LOS and costs are optimized with regards to teaching status should be done.

Categories: Orthopedics, Pulmonology, Quality Improvement

Keywords: copd, total hip arthroplasty, cost, mortality, length of stay, teaching status

\section{Introduction}

In the United States, total hip arthroplasty (THA) is a commonly performed procedure in orthopedic surgery, most often in the treatment of osteoarthritis, osteoporosis, avascular necrosis of the femoral head, proximal femoral head fracture, or rheumatoid arthritis [1]. The average age for patients to undergo their first primary THA is 66.6 years $(S D=10.2)$ [1]. The number of total hip arthroplasties performed in the United States for patients aged over 45 increased from 138,700 , with a rate of 142.2 performed per 100,000 people in the year 2000 , to 310,800 with a rate of 257 performed per 100,000 people in the year 2010 [2]. This trend is expected to continue in the near future, with an estimated 572,000 THAs performed by the year 2030 [3]. Regardless of its invasive nature, in general, THA is well-tolerated with relatively good outcomes [4].

Chronic obstructive pulmonary disease (COPD) is a common comorbidity with a prevalence of up to $12 \%$ of the population in key states and a national mortality rate of 39.1 per 100,000 in the United States [5]. COPD is the fourth leading cause of death in the United States and direct expenses related to the disease's treatment are expected to rise to $\$ 49$ billion by the year 2020 [6]. COPD care has previously been associated with anesthesia-related complications postoperatively and intraoperative or postoperative pulmonary complications [7]. It has also been shown to negatively impact outcomes in total hip arthroplasty, including 90-day mortality, one-year mortality, one-year wound infection, 30-day readmission for hospitalization, 30-day pneumonia, 30-day acute respiratory failure, 30-day cerebrovascular accident, and length of stay during hospitalization [8].

Teaching status in orthopedic surgery has also been shown to influence outcomes in THA [9]. However, among the studies that have examined the effect of COPD and orthopedic surgeries, the authors were unable to find any that included hospital teaching status as a tested variable. The purpose of this study was to examine the effect of hospital teaching status on procedure and care cost, hospital length of stay (LOS), mortality, and the number of chronic conditions in those undergoing elective THA with and without COPD.

\section{Materials And Methods}

\section{Database}

The National Inpatient Sample (NIS) is the largest, all-payer, publicly available inpatient care database in the United States and is part of the Healthcare Cost and Utilization Project (HCUP). It is a stratified 20\% sample of all patient discharges from HCUP-participating academic and non-academic hospitals in 38 states and contains information on over seven million hospital discharges per year. The NIS has been shown to be adequate for the estimation of data that is nationally representative [10].

\section{Sample selection}

Deidentified patient discharge data from 2012-2014 were acquired from the NIS database and adult patients ( $\geqslant 18$ years) undergoing elective total hip arthroplasty were identified using 


\section{Cureus}

International Classification of Disease 9th edition (ICD-9) procedure code(s). Patients with a principal procedure code of total hip arthroplasty (International Classification of Diseases, Ninth Revision, procedure 81.51) were identified. From this sample, patients with COPD ICD-9 codes, including chronic bronchitis (491) or emphysema (492), along with their secondary codes, were extracted as described in Table 1.

\section{ICD-9 Code ICD-9 Secondary Code}

Chronic Simple chronic bronchitis (491.0), Mucopurulent chronic bronchitis (491.1), Obstructive chronic bronchitis w/o exac Bronchitis (491.20), Obstructive chronic bronchitis w(ac) exac (491.21), Obstructive chronic bronchitis w ac bronc (491.22), (491) Chronic bronchitis NEC (491.8), Chronic bronchitis NOS (491.9)

Emphysema

\section{TABLE 1: ICD 9th Edition COPD Codes}

ICD: International Classification of Diseases, COPD: Chronic Obstructive Pulmonary Disease

This subset of patients was then stratified by the teaching status of the hospital, including urban teaching, urban non-teaching, and rural. For this study, a hospital was considered a teaching hospital if it had one or more Accreditation Council for Graduate Medical Education (ACGME)-approved residency programs, was a member of the Council of Teaching Hospitals $(\mathrm{COTH})$, or had a ratio of full-time equivalent interns and residents to beds of 0.25 or higher. Rural hospitals were not split according to teaching status, as rural teaching hospitals were rare [11]. Demographic and outcome data were extracted, including age at admission (years), length of hospital stay (days), total hospital charges (US dollars), number of chronic conditions, and mortality during hospitalization.

\section{Data analysis}

Data analysis was performed by one-way analysis of variance (ANOVA) with linear regression and chi-squared for significance values using SPSS software (IBM Corp., Armonk, NY, US). As the NIS samples constituted approximately $20 \%$ of United States hospital discharges, the results were multiplied by five in order to accurately represent the total population in the data. Ethical clearance and patient consent were not sought, as the NIS-HCUP database contains deidentified patient data. This study was determined to be Institutional Review Board (IRB) exempt. The statistical level of significance was decided at $\mathbf{\alpha}=0.05$.

\section{Results}

A total of 7,985 patients with COPD and 768,000 patients without COPD undergoing elective THA were identified.

\section{COPD vs. non-COPD}

Among all hospitals, COPD is associated with more costs, higher ages of admission, longer lengths of stay, and increased mortality when compared to patients who do not have COPD undergoing elective THA (Table 2). The average age for COPD patients who underwent elective THA was 68.24 years old (standard deviation (SD) of 10.8, 95\% confidence interval (CI) \pm 0.24 ). The average age for a non-COPD patient who underwent an elective THA was 64.92 years old 


\section{Cureus}

(SD of $11.9,95 \% \mathrm{CI} \pm 0.12$ ). The average cost of a COPD patient was $\$ 65,987$ (SD of $\$ 28,470$, $95 \% \mathrm{CI} \pm \$ 634$ ), and the average cost of a non-COPD patient was $\$ 55,585$ (SD $\$ 36,560,95 \% \mathrm{CI} \pm$ $\$ 82)$.

\begin{tabular}{|c|c|c|c|}
\hline Characteristic & COPD $(n=7,985)$ & Non-COPD $(n=768,000)$ & Significance Level \\
\hline Admission Age (years) & 68.24 & 64.92 & $p<0.001$ \\
\hline Cost (U.S. Dollars) & $\$ 65,978$ & $\$ 55,585$ & $p<0.001$ \\
\hline Length of Stay (Days) & 3.49 & 2.82 & $p<0.001$ \\
\hline Mortality & $0.7 \%$ & $0.1 \%$ & $p<0.001$ \\
\hline
\end{tabular}

\section{TABLE 2: COPD vs. non-COPD}

COPD: Chronic Obstructive Pulmonary Disease

\section{COPD and teaching status}

The analysis of the impact of COPD on THA with regards to teaching status yielded unexpected results (Table 3). Overall, the mortality rate was not significantly different between different teaching statuses $(p>0.5)$. From the data, most COPD patients undergoing elective THA had it performed at an urban teaching hospital $(n=4,280)$. The average age of admission for urban teaching, urban non-teaching, and rural hospitals was 65.7 years (SD 10.6, 95\% CI \pm 2.1 ), 68.2 years old (SD 10.2, 95\% CI \pm 0.2 ), and 67.2 years old (SD 11.1, 95\% CI \pm 0.8 ), respectively. The average inpatient cost was $\$ 59,174$ (SD $\$ 38,172,95 \% \mathrm{CI} \pm \$ 1,144$ ), $\$ 63,827$ (SD $\$ 33,280,95 \% \mathrm{CI}$ $\pm \$ 1,201$ ), and $\$ 56,141$ (SD $\$ 37,339,95 \% \mathrm{CI} \pm \$ 2,664$ ). The average length of stay was 3.47 days (SD 2.18, 95\% CI \pm 0.07 ) for urban teaching, 3.37 days (SD 2.37, 95\% CI \pm 1.09 ) for urban nonteaching, and 3.97 days (SD 2.97, 95\% CI \pm 0.21 ) for rural. The average number of chronic conditions was 7.58 (SD 2.86, 95\% CI \pm 0.09 ) for urban teaching, 7.07 (SD 2.75, 95\% CI \pm 0.10 ) for urban non-teaching, and 7.64 (SD 2.97, 95\% CI \pm 0.22 ) for rural.

\section{Characteristic}

Urban Teaching $(n=4280)$

Urban Non-Teaching $(n=2950)$

Rural ( $n=755)$

Admission Age (years)

67.53

68.20

67.28

Cost (U.S. dollars)

$\$ 59,174$

$\$ 63,827$

$\$ 56,141$

Length of Stay (days)

3.47

3.37

3.97

Chronic Conditions

7.58

7.07

7.64

\section{TABLE 3: COPD and Teaching Status}

COPD: Chronic Obstructive Pulmonary Disease 
When comparing urban hospitals by teaching status, there was no significant difference in LOS or average age of admission. When total costs and chronic conditions are examined, a counterintuitive trend is noted. For urban teaching hospitals, the number of chronic conditions was significantly higher $(\mathrm{p}<0.001)$ when compared to non-teaching hospitals. However, when total charges are compared, the data shows urban teaching hospitals costs are lower $(\mathrm{p}=0.016)$.

\section{Urban Teaching Vs. Rural}

Rural hospitals had longer lengths of stay when compared to urban teaching hospitals $(p=0.015)$. There was no difference between cost $(p=0.336)$, age of admission $(p=0.792)$, and number of chronic conditions $(\mathrm{p}=0.810$ ) between urban teaching and rural hospitals.

\section{Urban Non-Teaching Vs. Rural}

Between urban non-teaching and rural hospitals, there was no difference between age of admission ( $\mathrm{p}=0.334)$. Rural hospitals were associated with higher numbers of chronic conditions $(p=0.010)$ and longer lengths of stay $(p=0.0093)$. Despite this, total costs were significantly lower $(\mathrm{p}=0.013)$ as compared to urban non-teaching hospitals.

\section{Discussion}

Total hip arthroplasty (THA) is among the most common orthopedic surgical procedures performed in the United States and demand has risen dramatically over the last two decades, with estimates continuing to rise. One reason for THA being so common is that it drastically improves patient quality of life and function. Although largely beneficial to the patients, there are also notable associated risks with undergoing THA. Chronic obstructive pulmonary disease (COPD) is a major risk factor when undergoing any major surgical procedure, including THA. COPD has been found to be one of the most serious comorbidities in patients with osteoporosis, which is a common cause of THA [12]. With the potential for such serious complications, the connection between COPD and THA is imperative to both physicians and patients.

Consistent with the studies by Liao et al. and Yakubek et al., our study showed that patients with COPD had significantly higher mortality rates and length of stay (LOS) as compared to the non-COPD group $[8,13]$. Our goal, however, was to compare the differences between the results of THA in patients with COPD among teaching and non-teaching hospitals. Our data suggest that teaching hospitals have decreased costs for patients with COPD that undergo THA while also having a greater mean number of chronic conditions per patient. The natural assumption is that the teaching hospitals' spend is associated with higher costs. However, based on our data, this thought process is flawed in certain cases. The results of our study suggest that teaching hospitals are able to optimize care and be more efficient, which leads to lower costs to the patient. A study performed by Kowalik et al. showed that primary THA performed at teaching hospitals cost, on average, $\$ 47,514$ and $\$ 49,445$ at non-teaching hospitals and revision THA performed at teaching hospitals cost, on average, $\$ 67,820$ and $\$ 66,990$ at non-teaching hospitals [14]. COPD was not studied as a complicating comorbidity, but the higher cost of revision surgery in teaching hospitals may be due to an increased incidence of COPD among revision patients. The presence of resident physicians also leads to the potential of surgery with state-of-the-art procedures and equipment, which can decrease risk factors and cost. Likewise, rural hospitals, which are often assumed to have fewer resources, seem to be the most efficient at managing costs with regards to COPD patients undergoing THA.

Limitations to our study include the fact that teaching hospitals may or may not have residency programs with residents involved in the patient's direct THA or COPD care. In addition, 
hospitals within the teaching or non-teaching cohorts can have a wide array of services, surgical techniques, and levels of expertise from one individual facility to the next. Our sample size may not be large enough, especially for rural hospitals, to be adequately representative of the national norm.

\section{Conclusions}

The current study identified that, in contrast to previous studies, teaching hospitals have lower costs of care when treating patients undergoing THA with concurrent COPD when compared to urban non-teaching hospitals. This data suggest that teaching hospitals and rural hospitals are able to utilize healthcare in a cost-effective way, although the specific mechanisms of how teaching status affects costs are undoubtedly multifactorial. Likewise, despite longer lengths of stay and increased numbers of chronic conditions, rural hospitals have the lowest costs of all. Using this data, researchers can further examine the individual mechanisms and help diminish disparities among rural, teaching, and non-teaching hospitals. Our results may also aid surgeons and primary care providers in hospital selection or recommendations when considering THA in patients with COPD.

\section{Additional Information Disclosures}

Human subjects: All authors have confirmed that this study did not involve human participants or tissue. Animal subjects: All authors have confirmed that this study did not involve animal subjects or tissue. Conflicts of interest: In compliance with the ICMJE uniform disclosure form, all authors declare the following: Payment/services info: All authors have declared that no financial support was received from any organization for the submitted work. Financial relationships: All authors have declared that they have no financial relationships at present or within the previous three years with any organizations that might have an interest in the submitted work. Other relationships: All authors have declared that there are no other relationships or activities that could appear to have influenced the submitted work.

\section{Acknowledgements}

The authors would like to thank Andrew Brevik, MS, and Logan F. Hanson, DO, for their assistance with this publication.

\section{References}

1. Fourth AJRR annual report on hip and knee arthroplasty data . (2017). Accessed: January 20, 2019: http://ajrr.net/images/annual_reports/AJRR-2017-Annual-Report---Final.pdf.

2. Wolford ML, Palso K, Bercovitz A: Hospitalization for total hip replacement among inpatients aged 45 and over: United States, 2000-2010. NCHS Data Brief. 2015, 186:1-7. Accessed: January 20, 2019: https://www.cdc.gov/nchs/data/databriefs/db186.pdf.

3. Kurtz S: Projections of primary and revision hip and knee arthroplasty in the United States from 2005 to 2030. J Bone Joint Surg Am. 2007, 89:780-785.

4. Pinedo-Villanueva R, Turner D, Raftery J, Cooper C, Arden NK: Outcomes after total hip replacement. Osteoarthritis Cartilage. 2014, 22:s214. 10.1016/j.joca.2014.02.410

5. Zhang X, Holt JB, Lu H, Wheaton AG, Ford ES, Greenlund KJ, Croft JB: Multilevel regression and poststratification for small area estimation of population health outcomes: a case study of chronic obstructive pulmonary disease prevalence using BRFSS. Am J Epidemiol. 2014, 179:1025-1033. 10.1093/aje/kwu018

6. Guarascio AJ, Ray SM, Finch CK, Self TH: The clinical and economic burden of chronic obstructive pulmonary disease in the USA. Clinicoecon Outcomes. 2013, 5:235-245. 10.2147/CEOR.S34321

7. Lumb A, Biercamp C: Chronic obstructive pulmonary disease and anaesthesia . CEACCP. 2014, 


\section{Cureus}

14:1-5. 10.1093/bjaceaccp/mkt023

8. Yakubek GA, Curtis GL, Sodhi N, et al.: Chronic obstructive pulmonary disease is associated with short-term complications following total hip arthroplasty. J Arthroplasty. 2018, 33:19261929. 10.1016/j.arth.2017.12.043

9. Boylan MR, Perfetti DC, Naziri Q, Maheshwari AV, Paulino CB, Mont MA: Is orthopedic department teaching status associated with adverse outcomes of primary total hip arthroplasty?. J Arthroplasty. 2017, 32:S124-S127. 10.1016/j.arth.2017.03.003

10. Final report on calculating national inpatient sample (NIS) variances for data years 2012 and later. (2015). Accessed: December 4, 2018: https://www.hcupus.ahrq.gov/reports/methods/2015_09.jsp.

11. HCUP NRD description of data elements. Healthcare Cost and Utilization Project (HCUP). Agency for Healthcare Research and Quality. (2015). Accessed: April 1, 2019: http://www.hcup-us.ahrq.gov/db/vars/hosp_bedsize/nrdnote.jsp.

12. Anakwe RE, Middleton SD, Jenkins PJ, Butler AP, Aitken SA, Keating JF, Moran M: Total hip replacement in patients with hip fracture: a matched cohort study. J Trauma Acute Care Surg. 2012, 73:738-742. 10.1097/TA.0b013e3182569ee4

13. Liao $\mathrm{K}, \mathrm{Lu} \mathrm{H}$ : A national analysis of complications following total hip replacement in patients with chronic obstructive pulmonary disease. Medicine (Baltimore). 2016, 95:e3182. 10.1097/MD.0000000000003182

14. Kowalik TD, De Hart M, Gehling H, Gehling P, Schabel K, Duwelius P, Mirza A: The epidemiology of primary and revision total hip arthroplasty in teaching and nonteaching hospitals in the United States. J Am Acad Orthop Surg. 2016, 24:393-398. 10.5435/jaaos-d-1500596 